\title{
EVOLUÇÃO DOS DESEMBARQUES DE PESCADO EM ARACAJU, SERGIPE, BRASIL
}

\section{FISH LANDINGS DEVELOPMENTS IN ARACAJU, SERGIPE STATE, BRAZIL}

\author{
Rayane Menezes da Silva Bertho ${ }^{1}$, Ana Rosa da Rocha Araújo ${ }^{2}$, Maria Lúcia Góes de Araújo $^{3}$ \& \\ José Milton Barbosa ${ }^{4}$ \\ Departamento de Engenharia de Pesca e Aquicultura, Universidade Federal de Sergipe - UFS \\ *e-mail: anarosaaraujop@gmail.com
}

Recebido em 25 de abril de 2021 - Publicado em XX de junho de 2021

RESUMO As pescarias na costa sergipana geralmente são diárias e dependem das marés. O Terminal Pesqueiro de Aracaju (T.P. Aracaju) é o principal porto de desembarque pesqueiro do município de Aracaju, localizado no centro da cidade, e é responsável por $85 \%$ dos desembarques pesqueiros. Dados de volumes de desembarques pesqueiros são ferramentas importantes para a Gestão pesqueira incluindo espécies capturadas, tamanho da frota, artes de pesca utilizadas e comercialização. Portanto o objetivo geral desse estudo foi analisar os dados coletados no monitoramento nos anos de 2010 a 2016 no T.P. Aracaju observando a evolução dos desembarques de pescado. Os dados utilizados foram coletados pelo Projeto de Monitoramento dos Desembarques Pesqueiros - PMPDP especificamente os dados monitorados e registrados no Terminal pesqueiro de Aracaju. Os resultados indicaram que foram registrados desembarques de pescado de 92 embarcações, denominada localmente de lancha das quais 50 embarcações são originárias do T.P. Aracaju e as outras 42 embarcações originárias de outros municípios de Sergipe e de outros estados do Brasil. A produção total anual de pescado desembarcado foi de 426t (2010), 567t (2011), 504t (2012), 761t (2013), 910t (2014), 822 (2015) e 737 (2016). A receita anual variou de BRL 2.985.582 em 2010 a BRL 8.225.128 em 2016 demonstrando que a venda do pescado na primeira comercialização variou muito pouco nos três anos monitorados. Os resultados dão visibilidade da real produção desembarcada no T.P. Aracaju embasando políticas públicas de gestão pesqueira no estado de Sergipe.

Palavras-chave: desembarque pesqueiro, produção total, camarões-marinhos.
ABSTRACT Fishing on the Sergipe State coast is usually daily but depends on the tide. The main fishing landing port of the municipality of Aracaju is the Aracaju Fishing Terminal (T.P. Aracaju), located in the city center and responsible for $85 \%$ of the fishing landings. Data on fishing landings are essential tools for fisheries management, including caught species, fleet size, fishing gear and marketing. Therefore, this study's aim was to analyze the data collected in the monitoring years from 2010 until 2016 at the T.P. Aracaju observing the developments of fish landings. The Fisheries Landings Monitoring Project - PMPDP specifically monitored and recorded the data at the Terminal Pesqueiro de Aracaju. The results showed that during the monitoring years the landings of fish from 92 vessels, locally called "lancha", were recorded, of which 50 vessels originated from T.P. Aracaju and the other 42 vessels from other municipalities of Sergipe and other states of Brazil. The total annual production of landed fish was 426t in 2010, 567t in 2011, 504t in 2012, 761t in 2013, 910t in 2014, 822 in 2015 and 737 in 2016. Annual revenue ranged from BRL 2,985,582.00 in 2010 to BRL $8,225,128.7$ in 2016, demonstrating that the sale of fish varied little in the years monitored. The work's results show the real production landed in T.P. Aracaju, on which public policies of fisheries management in the state of Sergipe are based.

Key words: fishing landing, total production, marine shrimps. 


\section{Introdução}

A captura mundial de pescado, em 2018, alcançou 178,5 milhões de toneladas, sendo 54\% advindos das capturas em águas marinhas e continentais, e $46 \%$ da aquicultura (FAO, 2020). No Brasil, a produção de pescado apresentou tendência de crescimento na última década. Os últimos dados publicados referentes à estatística pesqueira nacional revelaram que a produção da pesca, em 2011, foi de 803.270 toneladas, com $68,9 \%$ desse total, oriundo da pesca em águas marinhas e $31,1 \%$ proveniente de águas continentais.

No Nordeste brasileiro foi registrada a maior produção de pescado capturado em águas marinhas, com 454.216,9 t, 31,7\% da produção nacional (MPA, 2011). A região foi responsável pela segunda maior produção pesqueira continental, com 68.700,9 t (MPA, 2011). No estado de Sergipe, segundo Thomé-Souza et al. (2013), a produção pesqueira em 2012 foi estimada em 4.787t e 2013 em 4001t. Em 2014 foi de 5.603t, sendo 3.629t advindos de áreas marinhas e 1.974t dos estuários (Araújo et al., 2016).

O litoral do estado de Sergipe possui ao longo dos seus $163 \mathrm{~km}$ de costa, cinco grandes estuários com uma biodiversidade aquática relevante (Seap/Ibama/Prozee, 2006). A capital Aracaju possui 35km de costa, banhado pelos Rios: Sergipe, Vaza Barris, Poxim, Rio do Sal e Pitanga (Almeida, 2008), o que torna ainda mais rico a diversidade dos organismos aquáticos, principalmente pela presença de manguezais. $\mathrm{O}$ município possui cerca de mais de 600 mil habitantes de acordo com o IBGE (2016), dos quais $2 \%$ dos habitantes tiram seu sustento da pesca.

No município de Aracaju a pesca estuarina é realizada por uma frota composta de canoas a remo e vela e motorizados que operam exclusivamente nos estuários e rios. As canoas são responsáveis por 14\% da produção total desembarcada no município, em dez pontos de desembarques: Areia Branca, bairro Industrial, Boca do Rio, Bugio, Coroa do Meio, Japãozinho, Lamarão, Mosqueiro, Santos Dumont e Soledade (ThoméSouza et al., 2013; Araujo et al., 2016).

Enquanto a pesca marinha é exercida por uma frota de embarcações que operam na captura de peixes demersais (plataforma e talude continentais), na captura de camarões, ao longo da costa. (Ibama, 2005) e pelágicos (tunídeos e afins) (Carvalho et al. 2015). As pescarias na costa sergipana geralmente são diárias e dependem das marés, havendo raras exceções em que são realizadas viagens, com alguns dias exercendo a atividade pesqueira (Seap/Ibama/Prozee, 2005).

O Terminal Pesqueiro de Aracaju (T.P. Aracaju) é o principal porto de desembarque pesqueiro do município de Aracaju. Está localizado no centro da cidade, e é responsável por $85 \%$ dos desembarques pesqueiros do município, onde ocorre vendas diretas, assim como exportação, levando a renda para diversas famílias (Thomé-Souza et al., 2013; Araujo et al., 2016).

Durante sete anos foram coletados diariamente registrados em formulários dados de desembarques pesqueiros no T.P. Aracaju desembarques, incluindo: volume desembarcado, espécies capturadas, tamanho da frota, arte de pesca utilizada e comercialização, ferramentas importantes que para gerar estatísticas confiáveis sobre as operações de pesca informações básicas capazes de subsidiar política pública de gestão dos estoques pesqueiros.

Portanto o objetivo desse estudo foi analisar os dados do desembarque pesqueiro durante os sete anos (2010 a 2016) no T.P. Aracaju, considerando sua evolução e a geração de subsídios para gestão da atividade pesqueira.

\section{Material e Métodos}

Os dados utilizados foram originários do Projeto de Monitoramento dos Desembarques Pesqueiros PMPDP registrados no T.P. Aracaju (Figura 1) que possui $1.385 \mathrm{~m}^{2}$ de área construída e compreende entreposto de recepção, comercialização e beneficiamento de pescado, cais, fábrica de gelo, refeitório, vestiários e sanitários, subestação, câmaras frigoríficas, unidade de comercialização atacadista de produtos da pesca, unidade administrativa, estação de tratamento de efluentes, pátio de caminhões e estacionamento de veículos.

Foram utilizados dados primários coletados no Terminal no período de 2010 a 2016 . O número de registros variou de acordo com os desembarques realizados no porto e a disponibilidade de informações fornecidas pelos pescadores.

As informações registradas no formulário foram: nome da embarcação, tipo de embarcação, data de saída, data de chegada, número de pescadores, dias de pesca, arte de pesca utilizada, volume desembarcado no T.P. Aracaju por espécies em quilo (kg) e/ou unidade (unid). 


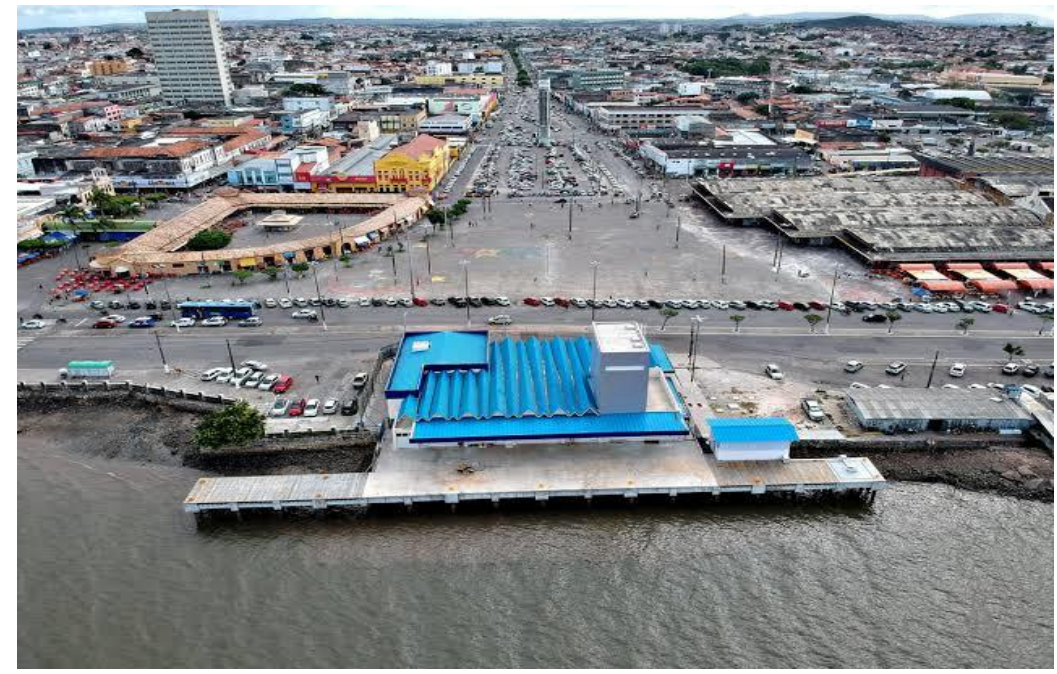

Figura 1. Terminal Pesqueiro de Aracaju (Fonte: camelempreendimentos.com.br/nossas_obras/construcao-do-terminalpesqueiro-publico).

Os volumes de desembarques foram registrados por espécies, com melhor detalhamento das mesmas ao longo dos sete anos. E a identificação das espécies capturadas foi realizada acordo com Barbosa, 2021.

A área de captura das embarcações monitoradas foi a zona costeira/marinha do litoral do estado de Sergipe (Figura 2), composta por 10 municípios ao longo de $163 \mathrm{~km}$, delimitados ao norte pelo rio São Francisco e ao sul pelo rio Real (Nascimento, 2011; Carvalho \& Fontes, 2006; 2007).

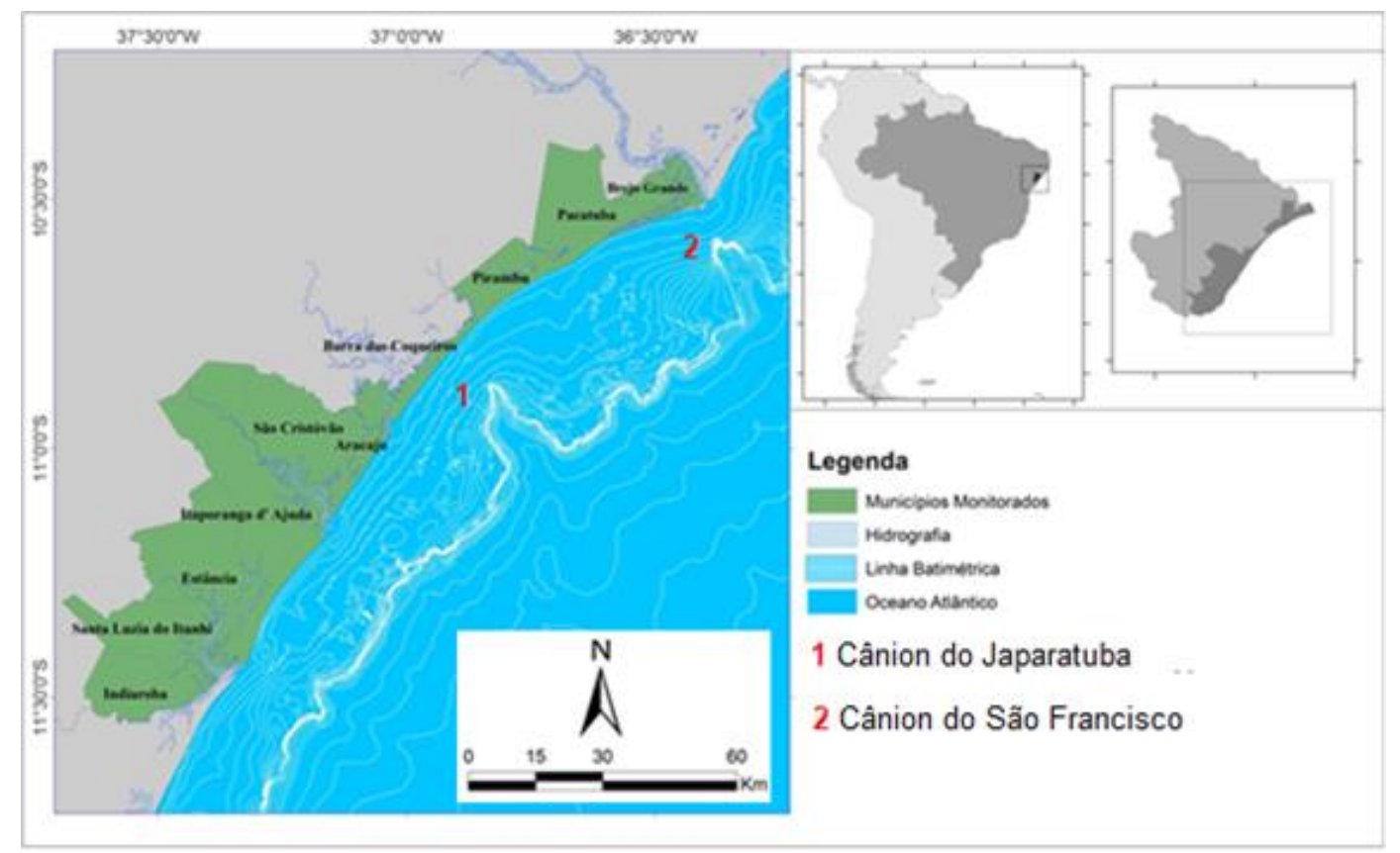

Figura 2. Área de atuação das embarcações monitoradas e que desembarcaram no Terminal Pesqueiro de Aracaju no período de 2010 a 2016.

A largura da plataforma continental varia de $8 \mathrm{~km}$ na cabeceira do cânion do São Francisco até $35 \mathrm{~km}$ ao norte do cânion do Japaratuba e a quebra da plataforma ocorre na profundidade de $50 \mathrm{~m}$, possuindo em geral uma baixa declividade (1:1000). A plataforma na área monitorada além da pouca largura possui ainda uma série de recortes por conta de dois canyons instalados em sua borda externa (Araújo \& Ribeiro, 2005; Guimarães, 2010; Aguiar et al., 2011; Figueiredo et al., 2011; Carneiro et al., 2017). 
Antes de iniciar o monitoramento dos desembarques pesqueiros foi realizado um diagnóstico sobre o número de embarcações existentes no T.P. Aracaju, identificando o nome da embarcação, proprietário, comprimento total, tipo de motor e tipo de embarcação. As embarcações que não foram registradas durante o diagnóstico e que realizaram desembarques foram cadastradas durante o monitoramento. Dessa forma foi obtido um banco de dados sobre as embarcações registradas no T.P. Aracaju e as que eram de outros portos. Para maior controle do número de barcos registrados no T.P. Aracaju, as informações obtidas no diagnóstico foram comparadas com os registros realizados nas Portarias Estadual, publicadas todos os nãos no diário do estado, e que solicitaram a isenção do óleo diesel.

A partir do controle das embarcações ativas foi realizada a extrapolação dos dados e estimados os volumes de desembarques $(\mathrm{Kg})$ e que são apresentados nesse estudo. Os dados foram extrapolados considerando: a média dos volumes totais de pescado desembarcado por arte de pesca e registrados multiplicados pelo número total de embarcações ativas.

Os dados foram extrapolados linearmente, conforme estabelecido na metodologia do Estatpesca (Aragão \& Castro e Silva, 2006), e estimadas as capturas totais de pescado anual desembarco no T.P. Aracaju utilizando o programa Excel na elaboração de gráficos.

\section{Resultados}

Durante os sete anos de monitoramento foram registrados desembarques de pescado de 92 embarcações, denominada localmente de Lancha, das quais 50 eram originárias de Aracaju e as outras 42 originárias de outros municípios de Sergipe e/ou de outros estados do Brasil.

A partir de 2012 foi observado um aumento nos desembarques de embarcações de outros locais, sendo que em 2014 foram registrados maiores desembarques de embarcações originárias de outros locais (36\%) no T.P. Aracaju, com diminuição nos anos seguintes monitorados, 2015 (19\%) e 2016 (20\%) (Figura 3).

As principais frotas que desembarcam pescado no T.P Aracaju são: a frota de arrasto duplo para camarões e a frota linheira dividida em: linha utilizada para peixes pelágicos e espinhel direcionado para captura de atuns e afins. A frota de arrasto duplo utiliza concomitantemente o corrico durante as pescarias de arrasto, o que propicia também a captura peixes pelágicos. A frota de espinhel era composta por embarcações originárias de outros estados: Bahia, Espírito Santo, etc. Durante o período de monitoramento foi observado que algumas embarcações originárias de Aracaju alternavam a pesca de camarão com arrasto duplo com pesca com linha ou espinhel direcionada para atuns e afins.

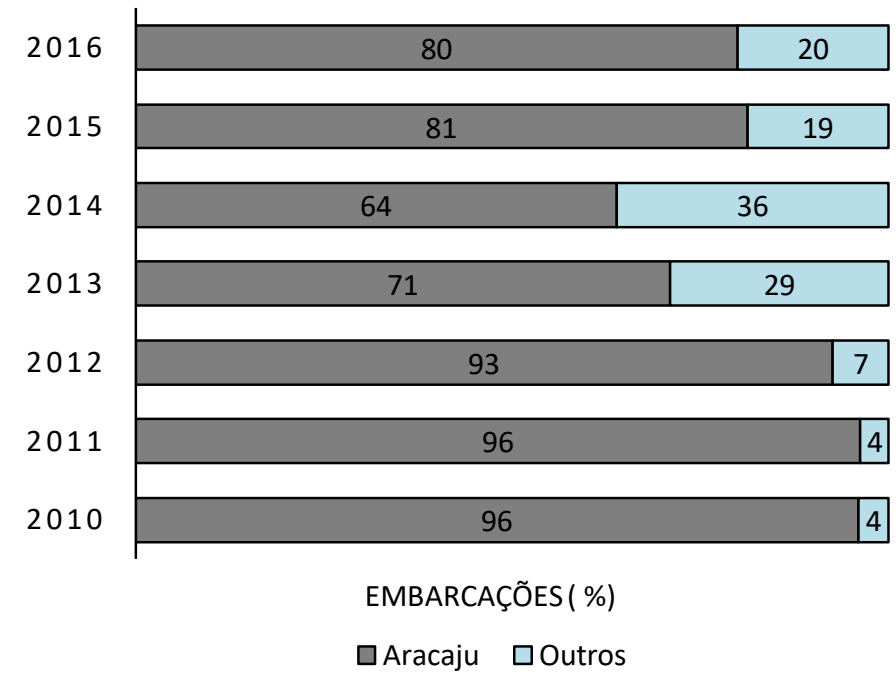

Figura 3. Porcentagem de desembarques da frota de lanchas no Terminal Pesqueiro de Aracaju, durante o período de 2010 a 2016.

As pescarias desembarcadas e monitoradas no T.P. Aracaju utilizaram arrasto duplo, espinhel e linha. A dinâmica da frota ao longo dos sete anos de monitoramento mostrou que a maioria dos desembarques de pescado no T.P. Aracaju foram provenientes de embarcações utilizando arrasto duplo. As pescarias com espinhel iniciaram desembarques somente em 2013 (Figura 4). 


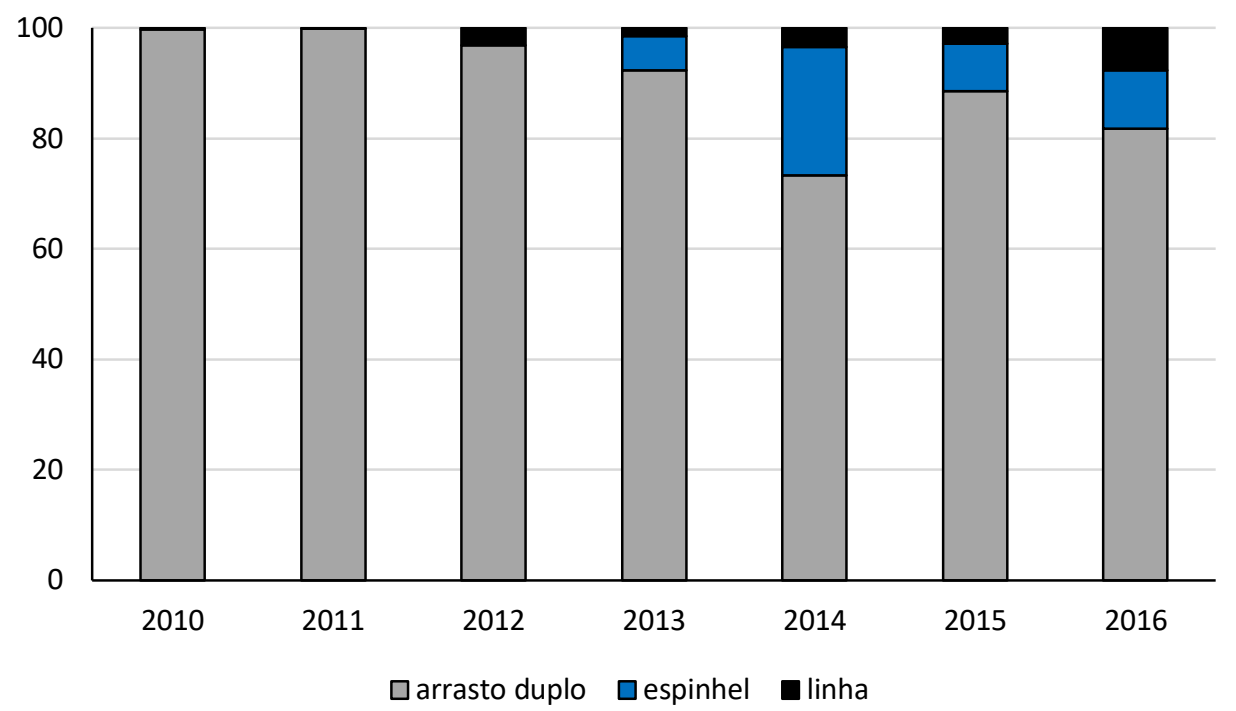

Figura 4. Artes de pesca utilizadas pela frota de lanchas que desembarcaram no Terminal Pesqueiro de Aracaju, durante o período de 2010 a 2016.

A área de pesca das embarcações, que utilizaram redes de arrasto duplo, foi a plataforma continental, onde a pesca com esse tipo de embarcação é direcionada para a captura de camarões, distribuída pelo ambiente costeiro raso e está associada às áreas de cascalho e lama, a uma profundidade que variou de 5 a 45 metros (Figura 5).

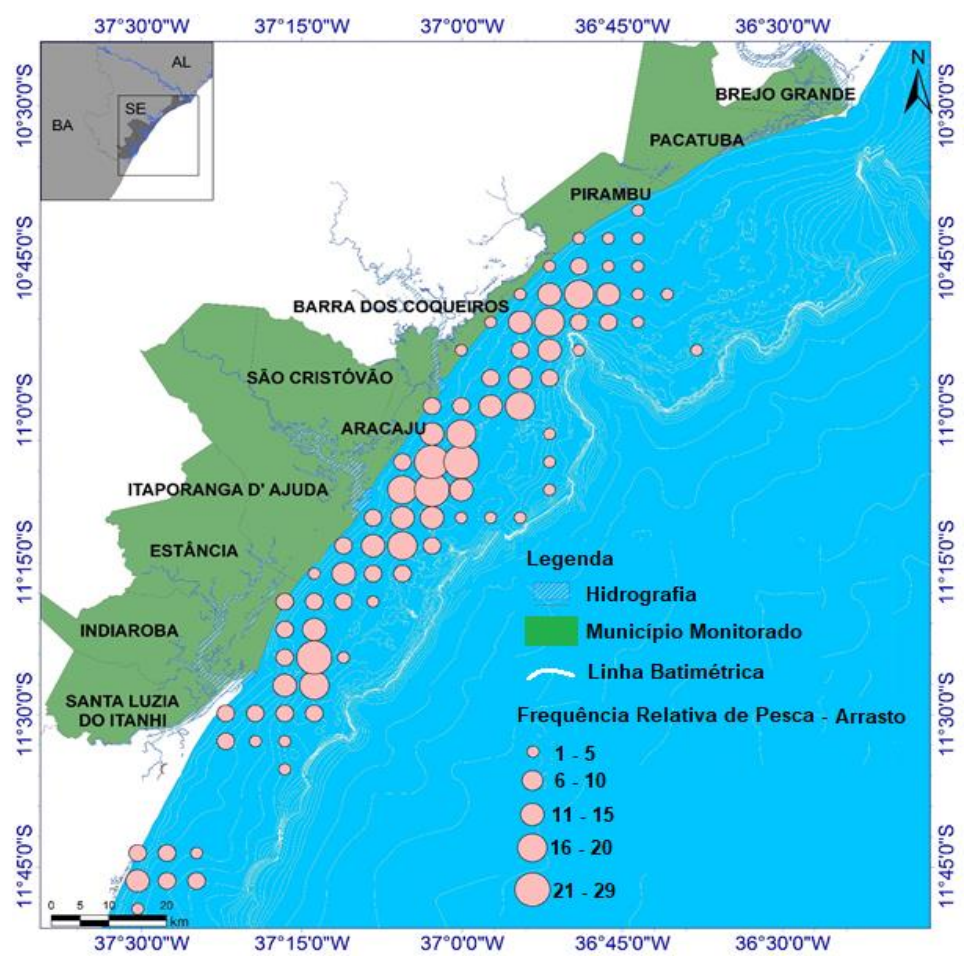

Figura 5. Frequência de eventos de pesca com rede de arrasto duplo no estado de Sergipe (quadrícula $=5 \mathrm{X} 5 \mathrm{~km}$ ).

As lanchas linheiras apresentaram frequência de distribuição em toda extensão marinha (principalmente no talude), com maior intensidade em frente ao estuário do rio Vaza Barris, com capturas direcionadas para peixes pelágicos (Figura 6). 


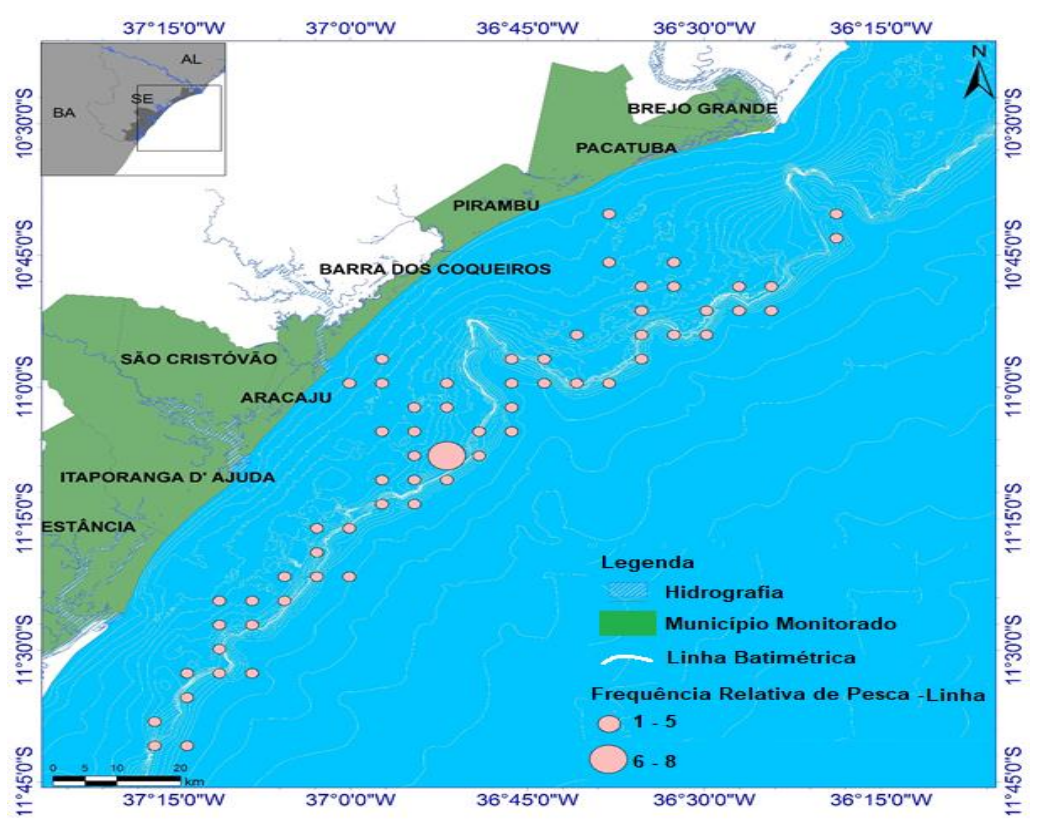

Figura 6. Frequência de eventos de pesca de lanchas linheiras no estado de Sergipe. (Quadrícula= 5 X 5 km).

As lanchas atuneiras apresentaram frequência de pescaria na zona oceânica, sendo que a maioria dos registros de pesca mais próximas do continente (no talude) foi direcionada para coleta de iscas vivas para posterior captura de atuns e afins (Figura 7). A pesca com esse tipo de embarcação está associada a estruturas submersas e semi-submersas, como as empregadas para a perfuração de poços ou produção/armazenamento de óleo e gás, ou zonas de encontro de massas de água, e predomina fortemente em zonas oceânicas.

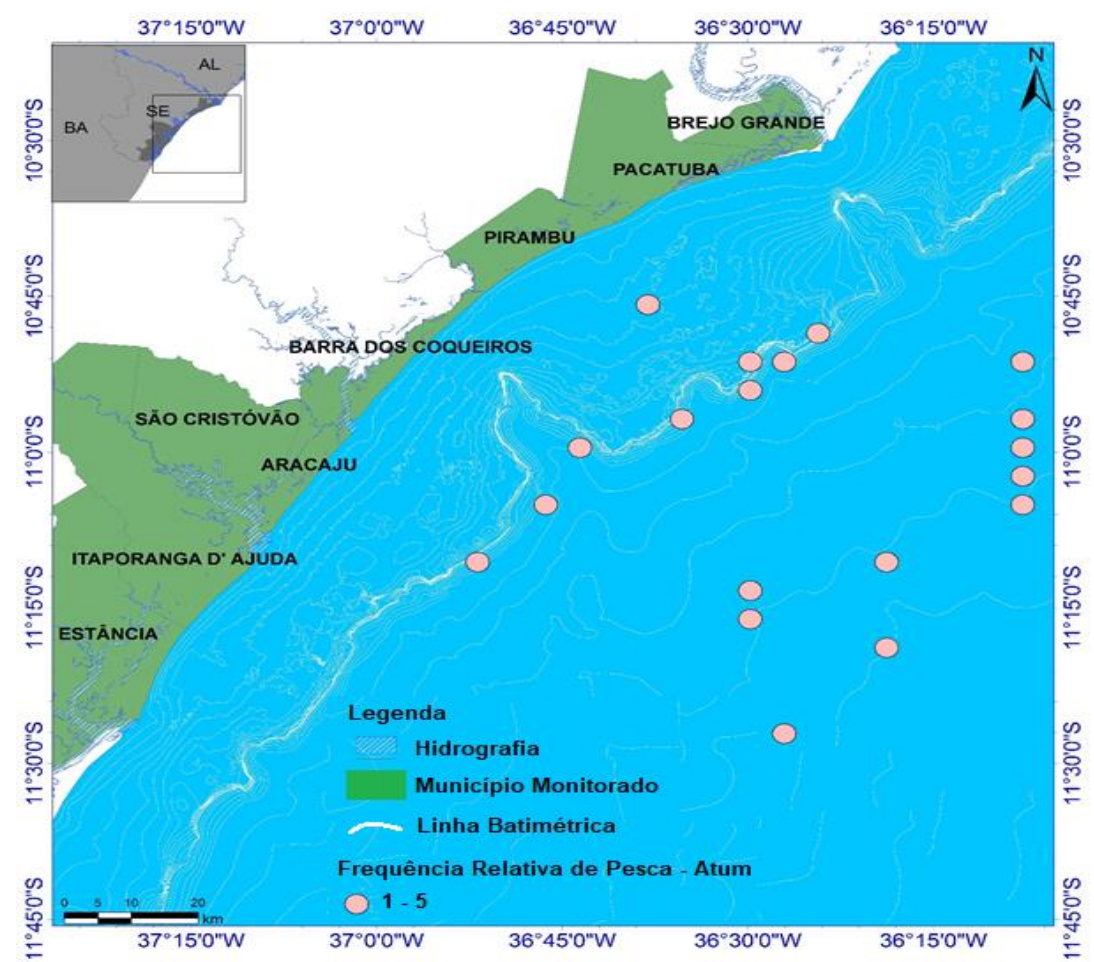

Figura 7. Frequência de eventos de pesca de lanchas atuneiras no estado de Sergipe (quadrícula $=5 \mathrm{X} 5 \mathrm{~km}$ ).

Todas as embarcações monitoradas ataram na zona costeiro-marinha do estado de Sergipe, as pescarias com arrasto duplo ocorreram em áreas rasas até 45 metros de profundidade e linhas e espinhéis em áreas mais profundas localizadas próximas a queda do talude (Figura 5, 6 e 7). 
A estimativa da produção total anual de pescado desembarcado variou ao longo dos sete anos monitorados, apresentando tendência de crescimento de 2010 até 2014, com maiores volumes de captura em 2014 (910 toneladas) e nos dois anos seguintes queda de 19\% em 2016 (Figura 8).

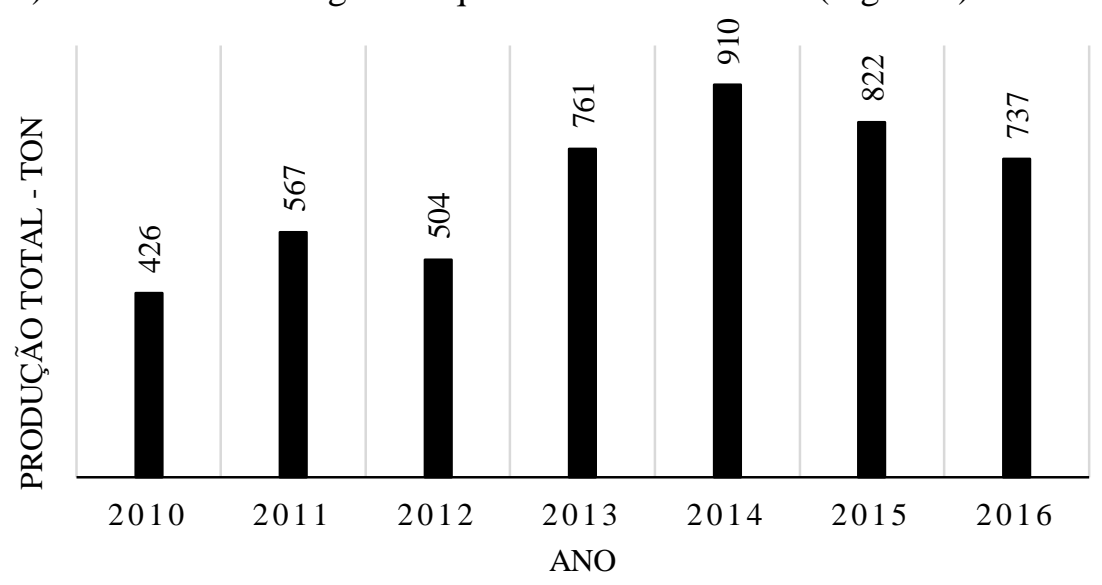

Figura 8. Estimativa da produção total anual de pescado desembarcado no Terminal Pesqueiro de Aracaju, durante o período de 2010 a 2016.

A estimativa da produção total por arte de pesca indicou que os maiores volumes de desembarques foram dos arrastos duplos e os menores foram com linha, em todos os anos monitorados.

Desembarques com espinhel foram registrados somente a partir de 2013 apresentando um pico em 2014 com capturas direcionadas para atuns e afins (Figura 9).

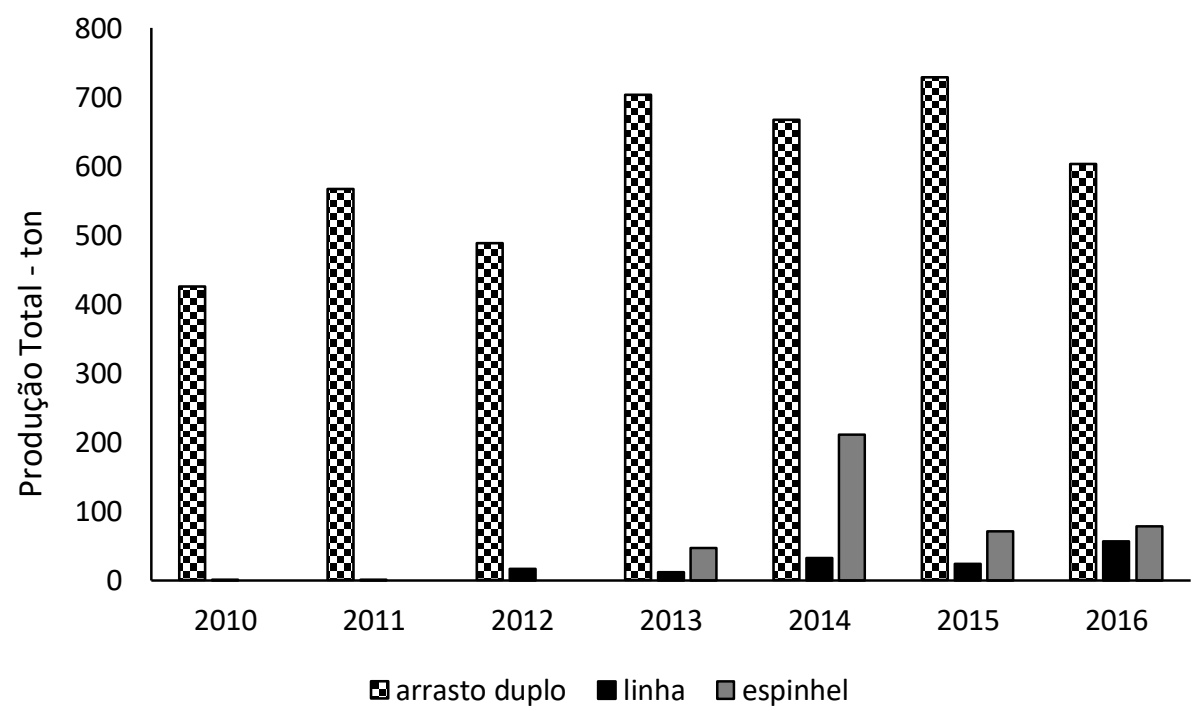

Figura 9. Estimativa da produção total em toneladas por arte de pesca, desembarcado no Terminal Pesqueiro de Aracaju, no período de 2010 a 2016.

As cinco principais espécies ou grupos em volumes de desembarques, que representaram $85 \%$ da produção total de Aracaju, foram: camarão-sete-barbas (Xiphopenaeus royeri), camarão-branco (Litopenaeus schimitt), camarão-rosa (Farfantepenaeus subtilis e Farfantepenaeus brasiliensis), atuns (Thunnus sp.) e pescadinha (Macrodon ancylodon). Analisando os volumes de desembarques dos camarões marinhos, em conjunto, foram registrados incremento de $43 \%$ no período de 2010 a 2013, com queda de $8 \%$ entre 2013 e 2014 e de $26 \%$ entre 2015 e 2016, período de flutuabilidade entre 2013 e 2016 (Figura 10). Os camarões marinhos representaram o principal pescado desembarcado no T.P. Aracaju com volumes de desembarques que atingiram o máximo de aproximadamente 572 toneladas ao ano, com destaque para as capturas de camarão-sete-barbas (60\%). 


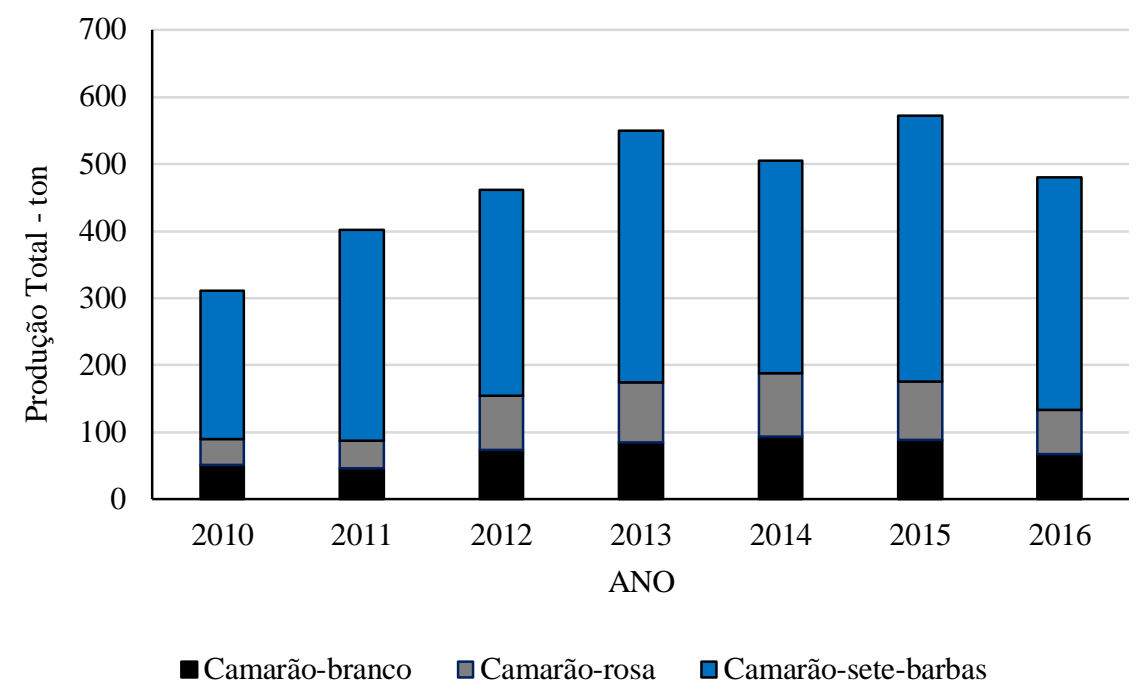

Figura 10. Estimativa da produção total anual de camarões marinhos desembarcado no Terminal Pesqueiro de Aracaju, durante o período de 2010-2016.

Os atuns apresentaram incremento de aproximadamente $200 \%$ entre 2010 e 2014 e queda de $70 \%$ entre 2014 e 2016 (Figura 11). E a pescadinha apresentou grande flutuabilidade com queda acentuada de aproximadamente 83\% em 2012 (Figura 12).

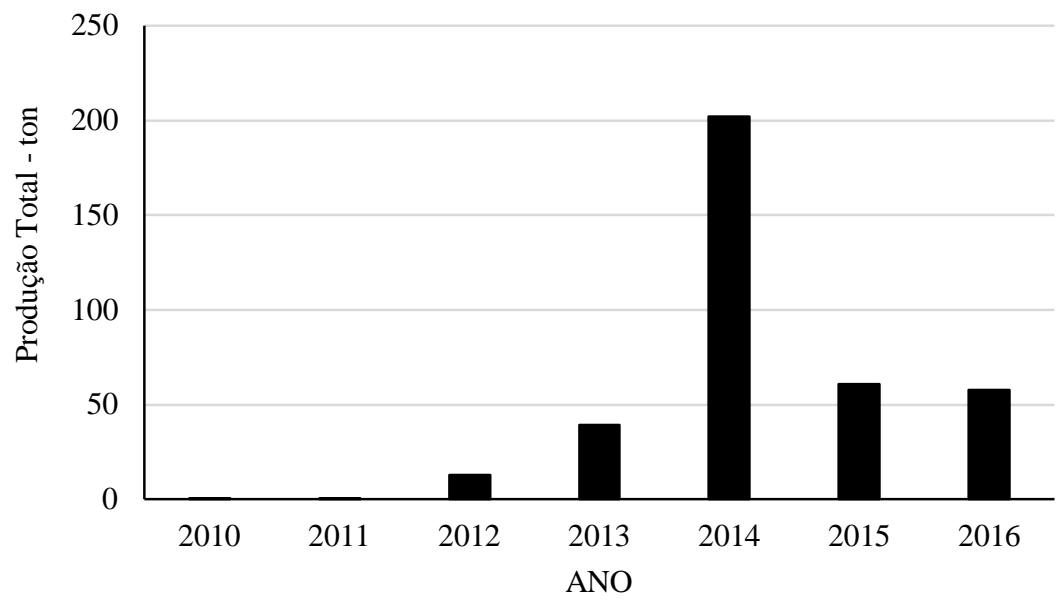

Figura 11. Estimativa da produção total anual de tunídeos e afins desembarcado no Terminal Pesqueiro de Aracaju, no período de 2010-2016.

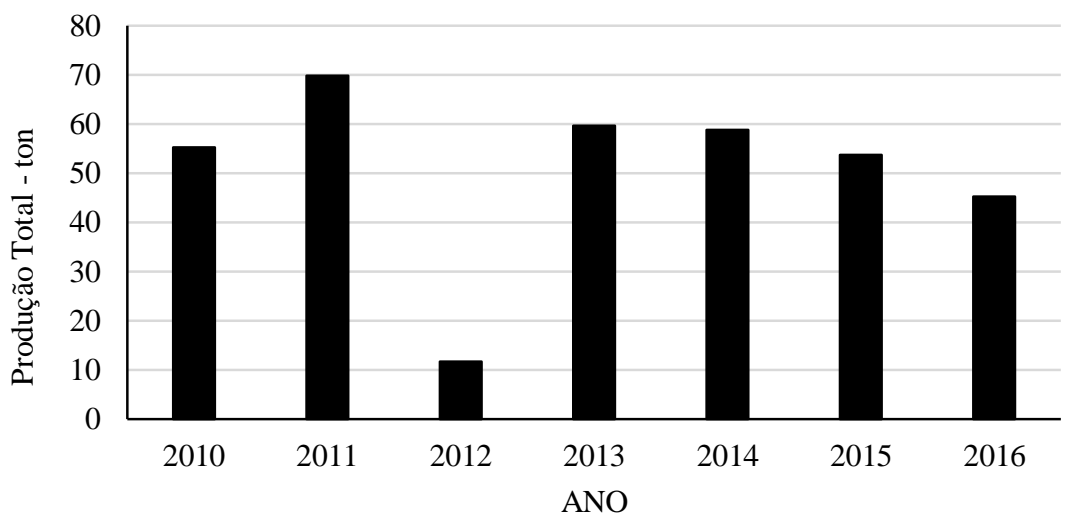

Figura 12. Estimativa da produção total anual de pescadinha desembarcado no Terminal Pesqueiro de Aracaju, durante o período de 2010 a 2016. 
O desembarque mensal das cinco principais espécies mostrou que os maiores volumes de captura ocorreram após o segundo período de defeso (de $1^{\circ}$ a 30 de abri), ou seja, o segundo semestre foi o mais produtivo (Figura 13). Ainda no gráfico é possível observar que no mês de abril, ainda por conta do defeso não tem a presença da pesca do arrasto e com isso uma pesca da pescadinha é nula.

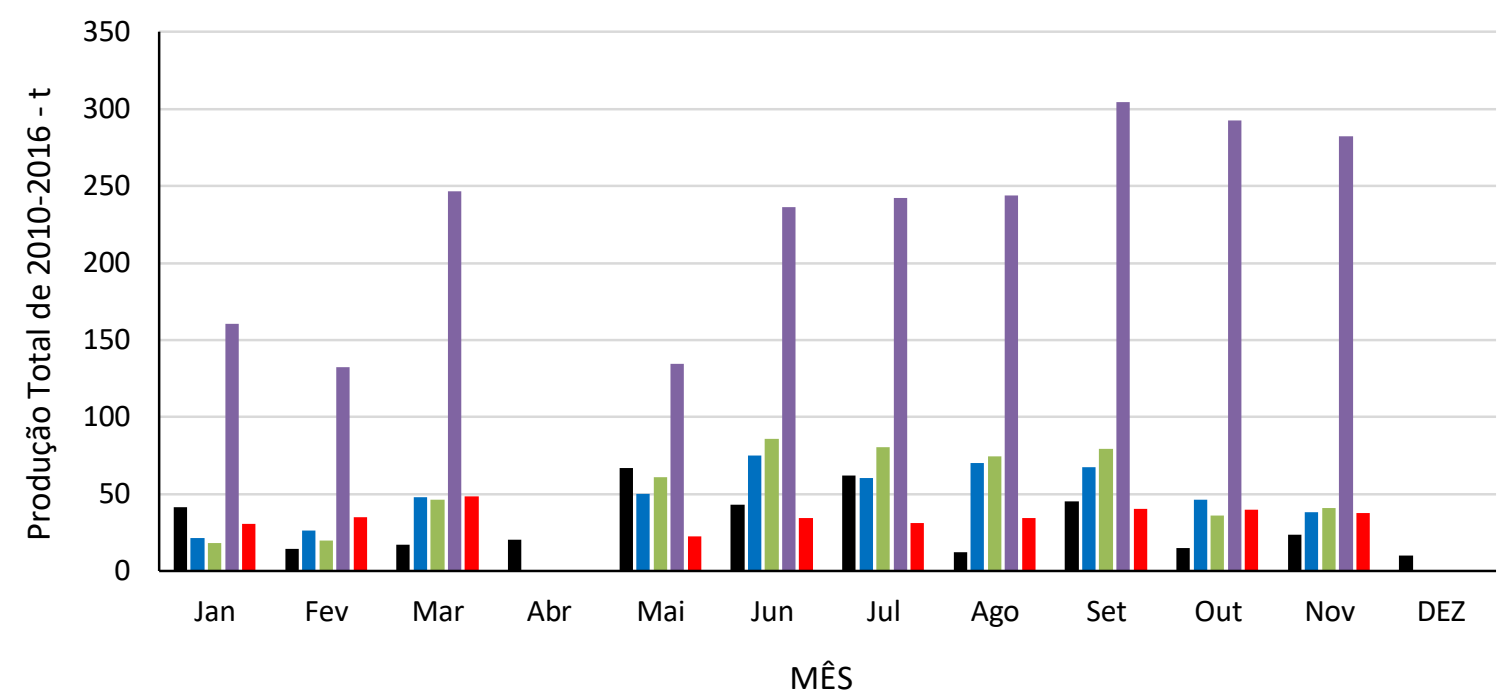

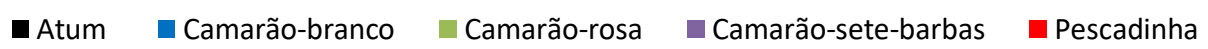

Figura 13. Produção total mensal das cinco principais espécies desembarcado no Terminal Pesqueiro de Aracaju, durante o período de 2010 a 2016.

O volume de pescado desembarcado movimentou um montante entre BRL 2.985.582,00 (2010) a 8.225.128,70 (2016) na primeira comercialização, a partir do T.P. Aracaju e apresentou crescimento entre 2010 e 2014, e déficit de $2 \%$ em 2016 (Figura 14).

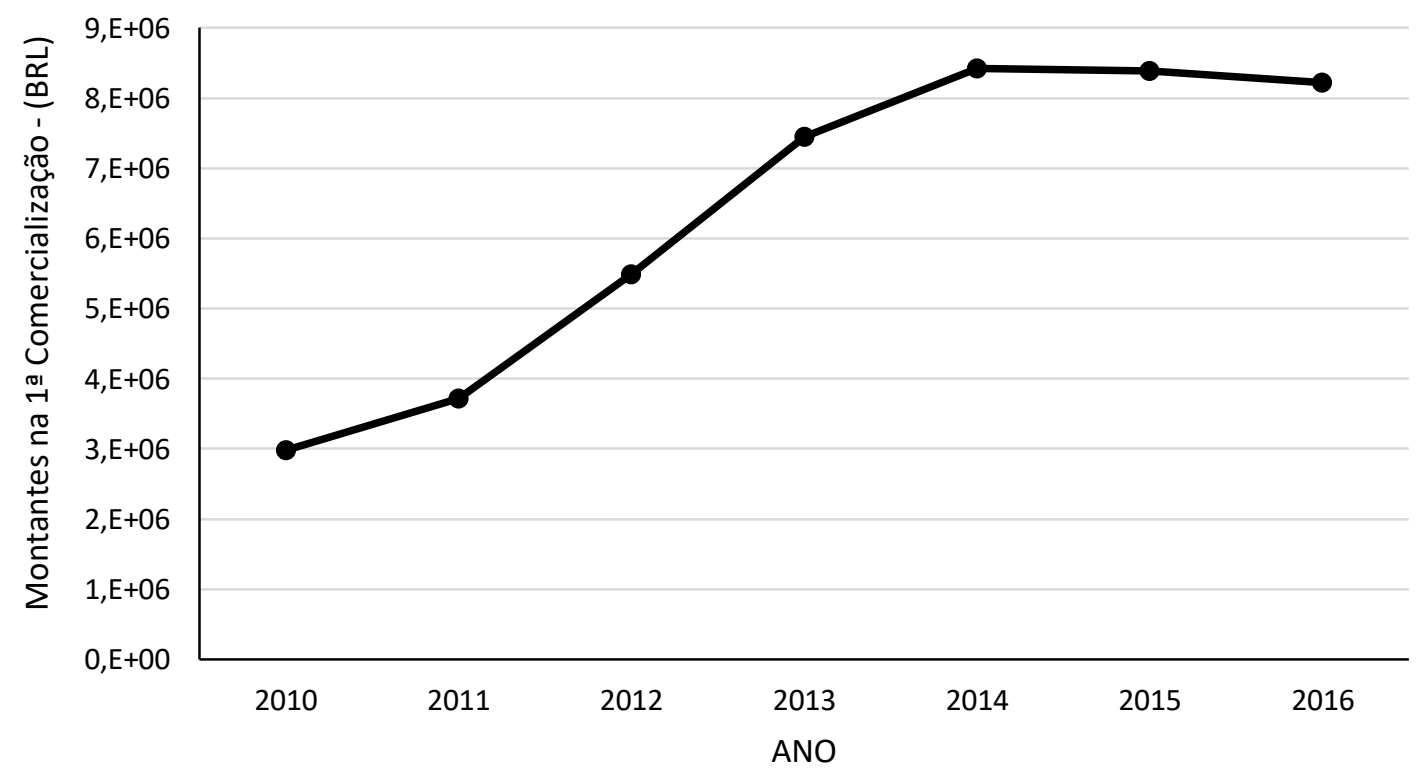

Figura 14. Montante movimentado em BRL na primeira comercialização de pescado entre os anos, durante o período de 2010 a 2016.

Considerando somente as pescarias de camarões com arrasto duplo a receita total foi composta pela venda de camarões incrementada pela venda de parte do bycatch comercializado (8\% da produção total) e pelas capturas com corrico (5\% da produção total). O bycatch é composto um volume expressivo de pescadinha e 
por uma grande diversidade de espécies, normalmente de pequeno porte, que foram reunidas sobre o nome de "Outros peixes".

Alguns grupos aparecem apenas quando ocorrem pescarias com espinhel, como por exemplo o agulhãomarinho e outros aparecem somente em alguns anos, por exemplo carapeba, corongo, etc. (Tabela 1).

Tabela 1. Estimativa da produção total de pescado por espécies, em quilo (kg), desembarcado no Terminal Pesqueiro de Aracaju, durante o período de 2010 a 2016.

\begin{tabular}{|c|c|c|c|c|c|c|c|}
\hline Espécies & 2.010 & 2.011 & 2.012 & 2.013 & 2.014 & 2.015 & 2.016 \\
\hline \multicolumn{8}{|l|}{ Peixes } \\
\hline${ }^{1}$ Agulhão-marinho & & & & 8.332 & 4.907 & 761 & 2.401 \\
\hline${ }^{2}$ Arabaiana & & & 725 & & 483 & 3.581 & 12.925 \\
\hline${ }^{3}$ Arraia & 55 & 347 & 806 & 10.151 & 13.681 & 11.418 & 8.782 \\
\hline${ }^{4}$ Atum (Albacoras) & 121 & 80 & 12.468 & 39.249 & 202.032 & 60.685 & 57.564 \\
\hline${ }^{5}$ Bagre & 185 & 685 & 151 & 288 & 565 & 355 & 237 \\
\hline${ }^{6}$ Bagre-guriaçu & & & & 33 & 73 & 21 & 292 \\
\hline${ }^{7}$ Beijupira & & & & & & & 37 \\
\hline${ }^{8}$ Bicuda/Barracuda & & & & 86 & 434 & 1.024 & 1.227 \\
\hline${ }^{9}$ Bonito & & & 390 & 127 & 7.823 & 4.462 & 2.164 \\
\hline${ }^{10}$ Cação & & & 367 & 4.517 & 3.080 & 1.738 & 10.590 \\
\hline${ }^{11}$ Camurupim & & & & & 31 & 88 & 136 \\
\hline${ }^{12}$ Cangulo & & & & & & & 288 \\
\hline${ }^{13}$ Caranha & & & & & 199 & & 343 \\
\hline${ }^{14}$ Carapeba & & & 28 & & & & \\
\hline${ }^{15}$ Catana & & & & & 55 & 202 & \\
\hline${ }^{16}$ Cavala & 372 & 963 & 364 & 1.494 & 2.986 & 4.651 & 8.125 \\
\hline${ }^{17}$ Corongo & & & & 30 & & & \\
\hline${ }^{18}$ Corvina & & & & 61 & 60 & 251 & \\
\hline${ }^{19}$ Dourado & & & 1.136 & 5.813 & 11.854 & 3.718 & 7.719 \\
\hline${ }^{20}$ Galo & 34 & 41 & & & 209 & 861 & 834 \\
\hline${ }^{21}$ Garacimbora & & & & & 15 & 796 & 450 \\
\hline${ }^{22}$ Garapau & & & & & 348 & 228 & \\
\hline${ }^{23}$ Gereba & & & & 44 & & & 26 \\
\hline${ }^{24}$ Guaiuba & & & & & & 165 & 1.082 \\
\hline${ }^{25}$ Guaricema & & & & 170 & 226 & 2.050 & 5.057 \\
\hline${ }^{26}$ Milongo & & & & & 92 & 381 & \\
\hline${ }^{27}$ Mirucaia & & & & & 241 & 982 & \\
\hline${ }^{28} \mathrm{Pampo}$ & & & & & 69 & 95 & \\
\hline${ }^{29}$ Paru & & 70 & & & 167 & & \\
\hline${ }^{30}$ Peixe Rei & 15 & 22 & & 25 & 664 & 938 & 1.341 \\
\hline${ }^{31}$ Pescada-amarela & & & 379 & & & 527 & \\
\hline${ }^{32}$ Pescada-branca & 241 & 788 & 220 & 3.384 & 1.091 & 1.335 & 428 \\
\hline${ }^{33}$ Pescada-bucu & & & 41 & & & 160 & 158 \\
\hline${ }^{34}$ Pescadinha & 55.196 & 69.929 & 11.712 & 59.717 & 58.751 & 53.782 & 45.189 \\
\hline${ }^{35}$ Robalo & & & & 25 & & 9 & \\
\hline${ }^{36}$ Roncador & & & & 38 & 61 & 7 & \\
\hline${ }^{37}$ Serra & 4 & 80 & & 295 & 5 & 276 & 323 \\
\hline${ }^{38}$ Sirigado & 47 & & & 57 & 2.293 & 1.959 & 4.374 \\
\hline${ }^{39}$ Solteira & 34 & & & & & 10 & \\
\hline
\end{tabular}




\begin{tabular}{lrrrrrrr}
\hline \hline & & & & & & & \\
\hline Tabela 1 (cont.) & 2.010 & 2.011 & 2.012 & 2.013 & 2.014 & 2.015 & 2.016 \\
\hline${ }^{40}$ Vermelha-Ariacó & 229 & 151 & 663 & 27 & 556 & 703 & 685 \\
${ }^{41}$ Vermelha-Cioba & 89 & & 117 & 16 & 640 & 141 & 1.284 \\
${ }^{42}$ Vermelha-Dentão & 96 & 116 & 96 & 51 & 1.857 & 898 & 2.370 \\
${ }^{43}$ Vermelha- olho-de-vidro & & & & & 906 & 4.916 & 9.811 \\
${ }^{44}$ Xaréu & 2.139 & 2.857 & 2.481 & 16.398 & 17.019 & 13.635 & 11.567 \\
${ }^{*}$ Outros peixes & 56.369 & 89.217 & 16.227 & 61.020 & 71.014 & 72.508 & 58.595 \\
\hline Crustáceos & & & & & & & \\
\hline${ }^{56}$ Camarão-branco & 51.620 & 45.990 & 72.113 & 85.109 & 93.610 & 88.743 & 67.727 \\
${ }^{46}$ Camarão-rosa & 38.723 & 41.449 & 79.733 & 88.843 & 93.934 & 87.176 & 66.104 \\
${ }^{47}$ Camarão-sete-barbas & 220.747 & 314.513 & 303.536 & 375.620 & 318.111 & 396.040 & 346.703 \\
${ }^{48}$ Siri-guajá & & & & 33 & & 32 & 81 \\
\hline Total geral & 426.316 & 567.298 & 503.753 & 761.053 & 910.142 & 822.308 & 737.019 \\
\hline
\end{tabular}

${ }^{1}$ Spp das famílias Istiophoridae e Xiphiidade; ${ }^{2}$ Spp do Gênero Seriola; ${ }^{3}$ Spp das famílias; $;{ }^{4}$ Spp do Gênero Thunnus; ${ }^{5}$ Spp da família Ariidae; ${ }^{6}$ Sciades proops $;{ }^{7}$ Rachycentron canadum; ${ }^{8}$ Spp do gênero Sphyraena; ${ }^{9}$ Katsuwonus pelamis e Auxis thazard; ${ }^{10}$ Spp das famílias Sphyrnidae e Carcharhinidae; ${ }^{11}$ Tarpon atlanticus: ${ }^{12}$ Balistes vetula e Aluteros monoceros; ${ }^{13}$ Lutjanus cyanopterus; ${ }^{14}$ Eugerres brasilianus; ${ }^{15}$ Trichiurus lepturus; ${ }^{16}$ Acanthocybium solandri e Scomberomorus cavala $;{ }^{17}$ Cynoponticus savana; ${ }^{18}$ Micropogonias furnieri; $;{ }^{19}$ Coryphaena hippurus $;{ }^{20}$ Selene spp; ${ }^{21}$ Caranx latus; ${ }^{22}$ Selar crumenophthalmus; ${ }^{23}$ Lobotes surinamensis; ${ }^{24}$ Ocyurus chrysurus; ${ }^{25}$ Caranx crysos; ${ }^{26}$ Lepophidium brevibarbe; ${ }^{27}$ Bardiella ronchus; ${ }^{28}$ Trachinotus spp; ${ }^{29}$ Chaetodipterus faber; ${ }^{30}$ Elagatis bipinnulata; ${ }^{31}$ Cynoscion acoupa; ${ }^{32}$ Cynoscion spp.; ${ }^{33}$ Cynoscion virescens; ${ }^{34}$ Macrodon ancylodon; ${ }^{35}$ Centropomus spp.; ${ }^{36}$ Conodon nobilis; ${ }^{37}$ Scomberomorus brasiliensis; ${ }^{38}$ Mycteroperca bonaci; ${ }^{39}$ Oligoplites saliens; ${ }^{40}$ Lutjanus synagris; ${ }^{41}$ Lutjanus analis; ${ }^{42}$ Lutjanus jocu; ${ }^{43}$ Lutjanus vivanus, Etelis oculatus e Lutjanus alexandrei; ${ }^{44}$ Caranx hippos; Camarão-branco

${ }^{45}$ Litopenaeus schmitti; ${ }^{46}$ Farfantepenaeus brasiliensis e F. subtilis; $;{ }^{47}$ Xiphopenaeus kroyeri; ${ }^{48}$ Calappa sp.

*Spp diversas provenientes do bycatch.

\section{Discussão}

O T.P. Aracaju é o principal porto de desembarque pesqueiro do estado de Sergipe e sua localização facilita a comercialização de pescado, em virtude de sua proximidade com o mercado público. A maioria das embarcações que desembarcam no referido terminal são de pequeno e médio porte com as mesmas características e modelo de construção, classificada como lanchas (Ibama, 2005). As principais diferenças estão relacionadas ao ano de construção, comprimento total e origem.

As embarcações originárias de Aracaju preferem desembarcar no T.P. de Aracaju pela existência estratégica de comercialização organizada a partir do mesmo, além disso, oferece logística de combustível e gelo facilitando a saída da próxima viagem. Durante o monitoramento não observamos aumento ou diminuição no número de embarcações registradas no T.P. Aracaju, a única mudança observada foi de venda e troca de nome de embarcações já existentes. Outras embarcações, de diferentes origens, frequentemente desembarcam nesse porto, porém não tem regularidade constante ou programada, a decisão de desembarcar no T.P. de Aracaju vai depender principalmente da logística de comercialização do pescado capturado por essas embarcações. A produção total anual desembarcada no T.P. Aracaju poderia ser muito maior, a estrutura física oferece condições para que mais embarcações possam desembarcar pescado aumentando o volume de pescado comercializado a partir desse porto.

A estimativa da produção total anual de pescado desembarcado variou ao longo dos sete anos monitorados, resultado associado principalmente com a quantidade de embarcações que desembarcam no T.P. Aracaju e a frota específica de espinhel com desembarques de atuns e afins. Os volumes de desembarques de camarões foram majoritários, maiores volumes de captura de camarões marinhos estão associados ao volume de chuvas, segundo declaração dos pescadores. Estudos tem relatados sobre as relações entre as capturas de camarões e a precipitação ou taxa de descarga de rios (Ives et al., 2009; Ayub, 2010). As capturas foram maiores após o segundo período de defeso, de 01 de abril a 15 de maio (primeiro de 01 de dezembro a 15 de janeiro), a pescaria é regulamentada pela proibição da pesca de camarões próxima a costa (menos de $3 \mathrm{mn}$ ) (Brasil, 2004; Ibama, 2011). 
O período de maiores volumes de capturas está entre junho e novembro, período de safra dos camarões marinhos e pescadinha. Na estrutura física do T.P. Aracaju existe uma área definida para o beneficiamento artesanal de camarões realizado por mulheres. O camarão pequeno ou muito quebrado é destinado para um grupo de mulheres que retiram a casca e cabeção dando origem a um produto denominado de filé de camarão, agregando valor ao produto e gerando trabalho e renda para as mulheres.

A área de captura de camarões ocorre dentro da plataforma continental de Sergipe, plataforma estreita e, portanto, a pesca é caracterizada como de "águas rasas", A espacialização da frota e o volume de capturada das espécies pela pesca foram influenciados pelo comportamento migratório das espécies (Branco, 2005; Heckler et al., 2013). A espécie X. kroyeri tem preferência por águas costeiras entre 5 e $27 \mathrm{~m}$ de profundidade, em locais com fundo de areia e lama e salinidade entre 9 e 36,5 e não utilizam os estuários para crescimento (Kensley, 1997; Castro et al. 2005; Costa et al. 2007).

As embarcações atuneiras mudaram de área quando houve diminuição dos navios sondas na zona marinha de Sergipe as quais serviam de atratores naturais para essas espécies. Até meados dos anos 2000 os atuns (albacoras) capturados em Sergipe eram alvo apenas da pesca artesanal, com uma produção reduzida e que não ultrapassava 25 t. O incremento das pescarias de atuns e afins mudou quando a partir de 2007 ocorreu o ingresso da frota atuneira advinda de Itaipava (ES) para operar no litoral sergipano devido à presença de navios sondas e das plataformas de petróleo que funcionavam como atratores facilitando a captura dos cardumes (Carvalho, 2015). No final de 2014 e durante todo ano de 2015 foi registrado uma diminuição de navios sondas e consequentemente de desembarques de atuns e afins pela frota atuneira no T.P. Aracaju.

Os dois primeiros anos do monitoramento (2010 e 2011) foram de ajuste da metodologia de coleta e mobilização para que os pescadores conhecessem melhor o destino dos resultados e dessa forma pudesse haver maior adesão com mais pescadores informando os volumes de desembarques. Os volumes de desembarques de camarões apresentaram aumento de 2010 a 2013, com oscilações nos anos seguintes. A pescadinha é a espécie da fauna acompanhante que apresenta maior volume de desembarque com valor de mercado para comercialização. Em 2012 observamos uma diminuição acentuada no volume de desembarque, esse resultado provavelmente está associado ao não registro de todos os desembarques, pelo fato do coletor não ter registrado nos formulários ou o próprio pescador não querer fornecer a informação.

Os produtos com maior valor comercial são os camarão-branco (Litopenaeus schimitt) e camarão-rosa (Farfantepenaeus brasiliensis), no entanto o maior volume de captura é do camarão-sete-barbas (Xiphopenaeus royeri).

As receitas advindas do pescado foram crescentes até 2014 apresentando queda de $2 \%$ em 2016, resultado relacionado diretamente com a diminuição no volume de captura e o valor de mercado praticado na primeira comercialização. A renda dessa pescaria é somada a captura e comercialização de peixes pelágicos capturados com corrico e a comercialização de parte da fauna acompanhante.

Os resultados do presente trabalho podem embasar políticas públicas de gestão pesqueira. Visto que a gestão estratégica da pesca deve definir objetivos de forma factível de serem alcançados.

A produção anual desembarcada no T.P. Aracaju é cerca de 800t, no entanto a estrutura física do porto oferece capacidade para aumento na capacidade de recepção de pescado através da otimização de mais desembarques. Essa estratégia, segundo Cochrane (2002), consiste na soma de todas as medidas de ordenamento voltadas para atingir os objetivos biológicos, ecológicos, econômicos e sociais que devem ser alvo da gestão pesqueira.

\section{Referências}

Aguiar, J.E., Marins, R.V. \& Almeida, M.D. (2011). Comparação de metodologias de digestão de sedimentos marinhos para caracterização da geoquímica de metais-traço na Plataforma Continental Nordeste Oriental Brasileira. Geochimica Brasiliensis. 21 (3): 304-323.

Almeida, B.J.M. \& Barbieri, E. (2008) Biodiversidade das aves do manguezal da 13 de Julho em Aracaju, Sergipe. O Mundo da Saúde. 32, (3): 317-328.

Aragão, J. A.N. \& Castro-Silva, S.M.M. (2006). Censo Estrutural da Pesca, coleta de dados e estimação de desembarque de pescado. Brasília: Ibama.

Araújo, A.R.R.; Barbosa, J.M.; Santos, J.P.; Carvalho, B.L.F.; Garcia-Filho, E.B.; Deda, M.S.; Silva, C.O. \& Chammas, M.A. (2016). Boletim estatístico da pesca nos litorais de Sergipe e extremo norte da Bahia 2014. São Cristóvão: Editora da Universidade Federal de Sergipe. 
Araújo, H.M.P. \& Ribeiro V.A. (2005). Distribuição das espécies de chaetognatha na plataforma continental de Sergipe e Alagoas. Brazilian Journal of Aquatic Science and Technology, 9, (1): 19-23.

Ayub, Z. (2010). Effect of temperature and rainfall as a component of climate change on fish and shrimp catch in Pakistan. The Journal of Transdisciplinary Environmental Studies, 9 (1): 1-10.

Barbosa, J.M. (2021). Atlas da macrofauna aquática dos estados de Sergipe e adjacentes. Fotografia Deda, M. Disponível em: www.atlasmacrofaunasergipe.com.

Barletta, M.; Barletta-Bergan, A.; Saint-Paul, U. \& Hubold, G. (2011) The role of salinity in structuring the fish assemblages in a tropical estuary. Journal of Fish Biology, 66: 45-72. 2005.

Brasil/MPA - Ministério da Pesca e Aquicultura (2010). Boletim Estatístico da Pesca e Aquicultura 2010, Brasília: MPA.

Brasil/MMA. 2004. Instrução Normativa do Ministério do Meio Ambiente, n. 14, de 14 de outubro de 2004. Proibi a pesca de camarões peneídeos nos estados de Alagoas, Sergipe e Bahia. Diário Oficial da República Federativa do Brasil, Brasília, DF, 15 de outubro de 2004. Seção 1, 199.

Carneiro, M.E.R., Fontes, L.C.S., Kowsmann, R.O. \& Puga-Bernabeu, A. (2017). In: Geologia $e$ geomorfologia da bacia de Sergipe-Alagoas. Carneiro, M.E.R., Fontes, L.C.S, Kowsmann, O.R., PugaBernabéu, A. (Org.). Petrobras/UFS. São Cristóvão: Editora da Universidade Federal de Sergipe.

Carvalho, M.E.S. \& Fontes, A.L. (2006). Estudo ambiental da zona costeira sergipana como subsídios ao ordenamento territorial. Revista Geonordeste, ano XVII, (2): 10-39.

Carvalho, M.E.S., Fontes, A.L. (2007). A carcinicultura no espaço litorâneo sergipano. Revista da Fapese. 3 (1): 87-112.

Carvalho, M.F., Barbosa, J.M., Araújo, A.R.R. \& Souza, J.M. (2015) Cadeia de comercialização de tunídeos no Estado de Sergipe, Brasil. Acta Fish Aquat. Res. 3(1):1-12.

Cochrane, K.L. (2002). A fishery manager's guidebook. Management measures and their application. FAO Fisheries Technical Paper No. 424. Rome: FAO.

FAO. (2020). FAO yearbook. Fishery and Aquaculture Statistics 2018/FAO. Rome: FAO

Figueiredo, A.V.A. \& Maroti, P.S. (2011). Bacia hidrográfica do rio Sergipe - significado, identidade e escolha de usos a partir da percepção dos membros do comitê (Gestão 2008-2010). Rede - Revista Eletrônica do Prodema, Fortaleza, 7 (2): 22-41.

Guimarães, C.R.P. (2010). Estrutura e dinâmica dos sedimentos superficiais e da fauna bêntica na plataforma continental de Sergipe (Tese de doutorado). Salvador (BA) Instituto de Geociências da Universidade Federal da Bahia.

Ibama /MMA (2005). Estatística da Pesca 2004 - Brasil. Brasília: Ibama.

Ibama - Instituto do Meio Ambiente e dos Recursos Naturais Renováveis (2011). In: Dias-Neto, J. (Org.). Proposta de Plano Nacional de Gestão Para o Uso Sustentável de Camarões Marinhos do Brasil. Série: Plano de Gestão Recursos Pesqueiros. Brasília: Ibama.

Ives, M.C., Scandol, J.P., Montgomery, S.S. \& Suthers, I.M. (2009). Modelling the possible effects of climate change on an Australian multi-fleet prawn fishery. Marine and Freshwater Research, 60(12) 12111222, doi:10.1071/MF07110.

Nascimento, A.A. (2011). Sedimentação holocênica na plataforma continental de Sergipe, Nordeste do Brasil. [Dissertação de Mestrado], Salvador (BA), Universidade Federal da Bahia.

Seap/Prozee/Ibama. (2005) Relatório final do Projeto de Monitoramento da atividade pesqueira no litoral do Brasil - Projeto Estatpesca. Brasília: Seap/Prozee/Ibama.

Thomé-Souza, M.J.F.; Deda, M.; Santos, J.P.; Carvalho, B.L.F.; Araujo, M.L.G.; Garciov-Filho, E.B.; Félix, D.C.F \& Santos, J.C. (2013) Estatística Pesqueira da Costa do Estado de Sergipe e Extremo Norte da Bahia 2011. São Cristóvão: Editora da Universidade Federal de Sergipe. 